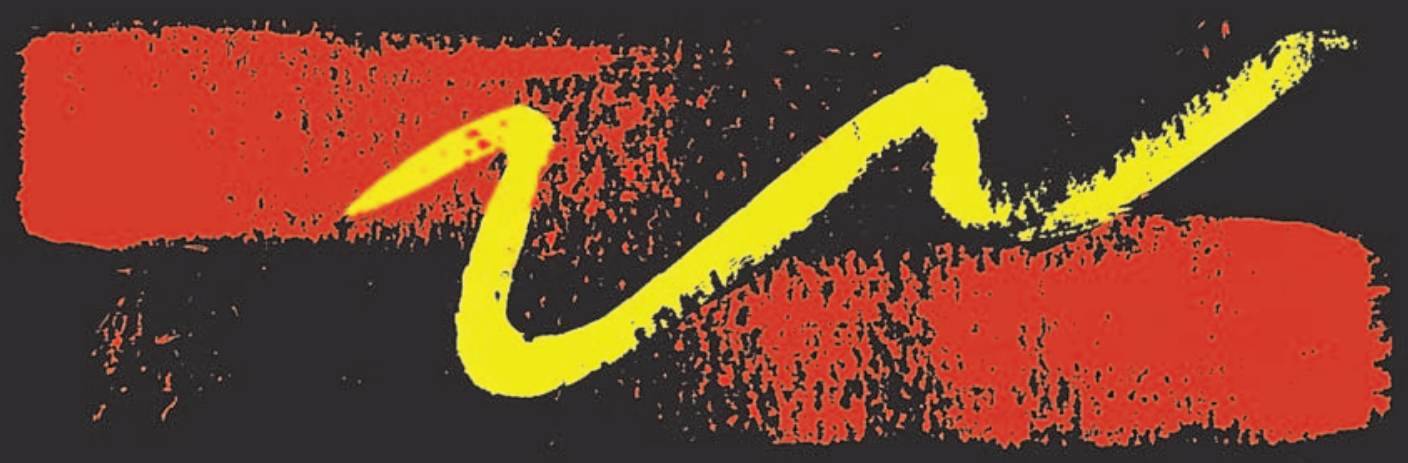

Vol. XXV (1), 2007
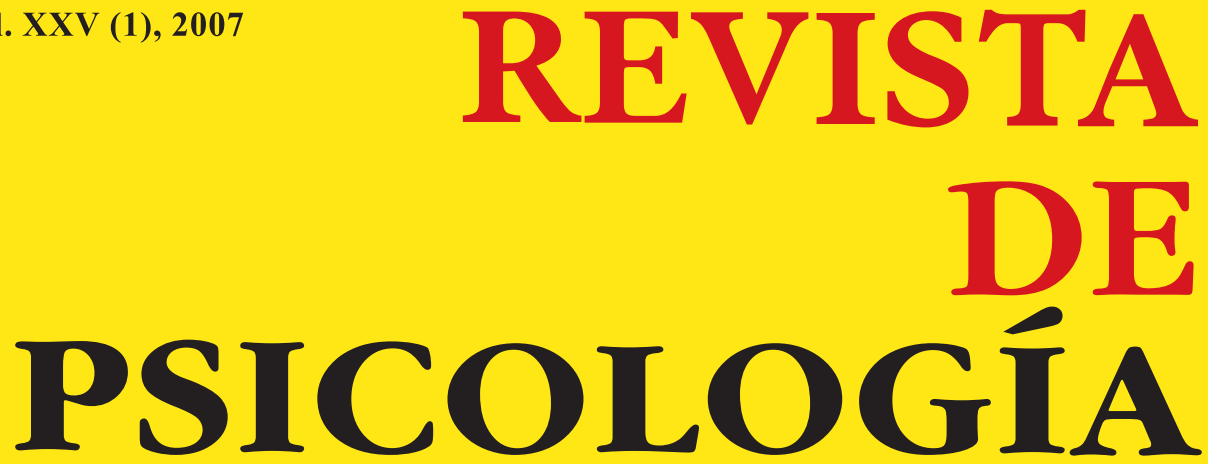

Sandra Castañeda

Anne Marie Costalat-Founeau

Daniel González

Jorge Haddad

Dirk Hermans

Carlos Iberico

Pierina Traverso

Debora Vansteenwegen

César Varela

Bram Vervliet 


\section{REVISTA DE PSICOLOGÍA \\ Vol. XXV (1), 2007}

\section{CONTENIDO}

\section{ARTÍCULOS}

Daniel González Lomelí, Sandra Castañeda Figueiras y César Varela Romero. Proceso de respuesta a examen de egreso en contabilidad: validación de constructo

Jorge Haddad Barthelemy. Experiencias y consideraciones en la conformación de perfiles de competencias

Pierina Traverso $K$. Dos madres adolescentes, dos vínculos: ¿qué marca la diferencia?

Carlos Iberico, Debora Vansteenwegen, Bram Vervliet y Dirk Hermans. El efecto de la (im)predictabilidad en el miedo contextual: una réplica de hallazgos básicos

Anne Marie Costalat-Founeau. Dinámica de la identidad, acción y contexto 
Revista de Psicología Vol. XXV (1), 2007 (ISSN 0254-9247)

\title{
Dos madres adolescentes, dos vínculos: ¿qué marca la diferencia?
}

\author{
Pierina Traverso K. ${ }^{1}$ \\ Pontificia Universidad Católica del Perú
}

\begin{abstract}
Se presenta un estudio de caso de dos madres adolescentes de la misma edad (17 años), con el cual se intenta explorar las representaciones maternas y las interacciones madre-bebe, es decir, las distintas maneras de "estar con" el bebe. El estudio pretende discutir que la maternidad adolescente en sí misma no es un factor de riesgo, sino que otras variables moderadoras como la calidad de las redes de sostenimiento y la función reflexiva con que cada joven madre cuenta. Las representaciones maternas fueron evaluadas a través de una entrevista semi-estructurada sobre representaciones maternas (Traverso, 2006) y las interacciones madre-bebe fueron medidas y filmadas a través del Mother-Infant Global-Ratings para dos y cuatro meses (Murray, Fiori-Cowley \& Cooper, 1996).

Palabras clave: maternidad adolescente, representaciones maternas, interacciones madrebebe, función reflexiva, redes de soporte.
\end{abstract}

\section{Two adolescent mothers, two bondings: What makes the difference?}

A case study of two adolescent mothers of the same age is presented (17 years old) with which it is intended to explore the maternal representations and mother-baby interactions, that is, the different ways of "being with" a baby. The study pretends to discuss that it is not adolescent motherhood in itself a risk factor, but other mediating variables such as support network and the reflective function with which each young mother counts. Maternal representations were assessed through a semi-structured interview about maternal representations (Traverso, 2006) and the mother-infant interactions were measured and filmed through the Mother-Infant Global Ratings for two and four months (Murray, Fiori-Cowley \& Cooper, 1996).

Key words: adolescent motherhood, maternal representations, mother-baby interactions, reflexive function, support network.

1 Doctora en Psicología por la Katholieke Universiteit Leuven, Bélgica. Profesora asociada del Departamento de Psicología de la PUCP. Se desempeña también como psicoterapeuta en la práctica privada. Dirección postal: Departamento de Psicología, PUCP, Av. Universitaria 1801, Lima 32, Perú. Correo electrónico: ptraver@pucp.edu.pe 

A partir de un estudio más amplio (Traverso, 2006) en que se investigaron las representaciones maternales e interacciones madre-bebe entre madres adolescentes y no adolescentes en un contexto de pobreza en Lima, Perú, se plantea para este trabajo el estudio de dos casos — procedentes de la misma muestra del estudio previo- con madres adolescentes de la misma edad (17 años), con representaciones maternas e interacciones madre-infante distintas entre sí: por un lado, una joven con una capacidad para "leer" e "interpretar" a su bebe, lo que se evidencia tanto en sus representaciones como en sus interacciones, y otra joven con dificultades para "pensar" en su bebe como un "otro" diferente de ella. No pretendemos polarizar a estas dos madres, es decir, presentar a una como adecuada y a otra como inadecuada; más bien queremos enfatizar en las diferentes maneras que tiene una madre - especialmente una madre adolescente- para acercarse a su bebe.

Este trabajo pretende dar cuenta de que no es la maternidad adolescente en sí misma un factor de riesgo para el vínculo madre-bebe como generalmente se piensa, sino que se trata de un conjunto de variables moderadoras $^{2}$ que rodean a la madre adolescente en contextos de pobreza que potencialmente pueden crear un factor de riesgo. Entre las múltiples variables moderadoras discutiremos dos: el uso de la función reflexiva, que se entiende como la capacidad para leer los estados mentales de uno mismo y de los demás (Fonagy, Gergely, Jurist \& Target, 2002), y el circulo protector de redes de soporte que rodean a la madre adolescente.

Para entender lo que son las representaciones maternas, tomamos la perspectiva de Daniel Stern (1997), quien define este concepto como

2 Se entiende como variable moderadora cualquiera que una otras dos o más variables, o que de alguna manera afecte la relación entre ellas. 
las construcciones mentales subjetivas que hacen las madres y que están organizadas en torno de la función maternal y del bebe. Estas incluyen experiencias actuales con el bebe, así como fantasías, miedos, sueños, memorias, profecías sobre el bebe, y sus propios modelos parentales. Estas representaciones son construidas sobre la base de la calidad de la experiencia interactiva con otros significativos y pueden influir en la conducta. Son modelos de estar con otro. Otra variable de nuestro estudio es la interacción madre-bebe, definida como un efecto recíproco de la conducta que cada uno de los miembros tiene sobre la conducta del otro.

Ser adolescente y ser madre al mismo tiempo es una situación vivida por el 10,7\% de las adolescentes del Perú y por el 5,7\% de las adolescentes de Lima (INEI, 2001). En el distrito de Villa El Salvador - lugar de donde provienen nuestras madres - existen aproximadamente 3,427 adolescentes que son madres, lo que representa el 11,27\% del total de adolescentes del distrito (Abad, 2003). Asimismo, las cifras absolutas del número de madres adolescentes en nuestro país ha ido incrementándose en los últimos años (INEI, 2001).

Por otro lado, la maternidad en adolescentes de nuestro país es una situación asociada con condiciones económicas precarias. Así, el 70,5\% de las madres adolescentes viven en situación de pobreza (Buitrón, 2003). La mayor parte de estas madres adolescentes provienen de las áreas más pobres y de estructuras familiares en las que la violencia, el abuso y la inestabilidad parental son predominantes (Contreras, 2001). Asimismo, Contreras (2001) encontró en su grupo de estudio que, del $54 \%$ de madres adolescentes que viven con sus parejas, solo el 3\% está casada. Del $46 \%$ que se mantienen solas, por lo menos la mitad de ellas conservan algún tipo de contacto con el padre biológico del bebe.

Esta asociación de maternidad en adolescentes y pobreza es más evidente en un distrito como Villa El Salvador, donde el 48,4\% de los hogares tiene al menos una necesidad básica insatisfecha y el 18\% de 
las viviendas son hacinadas (Abad, 2003). El 63\% de las jóvenes madres no tienen educación o solo han terminado estudios primarios (INEI, 2001). En términos generales, en este distrito un $50 \%$ de los habitantes vive en condiciones de pobreza, mientras que el $22.7 \%$ vive en condiciones de extrema pobreza (Apoyo Opinión \& Mercado, 2004). A través de los años, Villa El Salvador ha sido reconocida con varios premios internacionales por la promoción de la cultura, educación popular y organización comunal (Franco, 1993). Especialmente importante ha sido la participación de las mujeres en el fomento y la creación de estas organizaciones (Pimentel, 2000).

En la literatura psicológica y psicoanalítica encontramos dos perspectivas teóricas frente a la maternidad adolescente. El primer enfoque teórico (Koniak-Griffin \& Turner-Pluta, 2001; Luster \& Mittelstaedt, 1993; Stevens-Simon \& Mc Anarney, 1996) sería el de postular que la maternidad adolescente es una conducta de riesgo en la medida en que interrumpe el proceso de separación e individuación propio de la adolescencia, en especial respecto de la propia madre. Estas jóvenes, además, tendrían dificultades para lograr sus metas educacionales, lo cual disminuye las posibilidades de acceder a buenas oportunidades profesionales. Asimismo, se ha observado que estas jóvenes tienen un mayor número de hijos, lo cual a la larga incrementa sus niveles de pobreza.

Un segundo enfoque (Musick, 1994; Osofsky, Hann \& Peebles, 1993; Smithbattle, 2000) refiere que, a pesar de las dificultades inherentes a la tarea de la maternidad, encontramos que esta puede tener un rol estructurante en la identidad de las jóvenes, con lo que se convierte en una conducta resiliente. Las madres son capaces de reinterpretar las consecuencias negativas de la maternidad al otorgarles un significado positivo; de esta manera, es considerada un momento de cambio y transformación positiva (Nóblega, 2006). La maternidad, para estas jóvenes, sería también una forma de identificación con sus propias madres y una confirmación de su feminidad. 


\section{Investigaciones realizadas con madres adolescentes y sus bebes}

Encontramos que los resultados de las investigaciones con madres adolescentes y sus bebes no son siempre consistentes. Se podría decir que las madres no son un grupo homogéneo. Esto nos lleva a pensar que hay variables moderadoras - como mencionamos anteriormente- que influyen en las diferencias de los resultados obtenidos por los diferentes estudios.

En investigaciones realizadas en otros contextos sociales, se ha encontrado que los niños de madres adolescentes presentan un apego inseguro donde predominan los patrones evitativos y desorganizados (Osofsky, Hann \& Peebles, 1993). Luster y Dubow (1993) reportaron que los niños de madres adolescentes expuestos a diferentes factores de riesgo eran más propensos a presentar problemas cognitivos y socioemocionales en comparación con otros niños.

Las madres adolescentes son también más restrictivas y menos flexibles que las madres mayores. Su estilo de interacción en el juego es intrusivo, menos recíproco, así como menos involucrado y más controlador. Ellas están más focalizadas en sí mismas que en los bebes. Pueden usar el castigo físico y ejercer conductas hostiles hacia sus niños —un riesgo potencial de maltrato-. Pueden también ser muy críticas con sus hijos y mostrar poca tolerancia a la conducta exploratoria del niño. Las madres adolescentes tienen, asimismo, expectativas poco realistas con sus niños: pueden esperar mucho o muy poco de ellos. Estas expectativas, por tanto, pueden tener una influencia negativa sobre las interacciones. Las jóvenes madres y sus hijos entran en patrones irregulares de interacción afectiva; esto quiere decir que pueden predominar los afectos negativos o que las señales afectivas pueden ser mal interpretadas por la díada (tal como fue revisado por Brooks-Gunn \& Chase Lansdale, 1994; Luster \& Mittelstaedt, 1993; Musick, 1994; Osofsky, Hann \& Peebles, 1993). 


\section{Soporte social}

El soporte instrumental y emocional puede ser un factor protector para estas jóvenes madres, especialmente en poblaciones de bajo nivel socio-económico. En un estudio con madres adolescentes latinas, Contreras, Mangelsdorf, Rodees, Diener y Brunson (1999) encontraron que aquellas que tenían mayor soporte social eran más expresivas y sensitivas con sus bebes. No se trata de un soporte específico - ya sea por parte de la madre, la pareja o los amigos-, sino un soporte general que la cultura promueve, por ejemplo, al establecer sus hogares en la misma vecindad que la de sus padres — como en el caso de las madres estudiadas-.

Existen muchos y diferentes estudios sobre la relación de la madre con su propia madre. Sin embargo, en términos generales, se concluye que lo que más ayuda a una madre adolescente es que pueda adquirir autonomía con respecto a su propia madre, a la vez que se mantenga una adecuada cercanía emocional entre ellas (Wakschlag, Chase-Lansdale \& Brooks-Gunn como se cita en Levine Coley \& Chase-Lansdale, 1998). Este vínculo así descrito obviamente depende en gran medida de la historia entre madre e hija previa al embarazo. En ese sentido, Becerra (1995), en un estudio con adolescentes embarazadas de sectores pobres de Lima, encontró que una relación conflictiva entre madre e hija constituía el más frecuente precursor del embarazo adolescente.

En cuanto a la figura de la pareja, Samuels, Stockdale y Crase (1994) refieren que el soporte brindado por el padre del bebe mejora el nivel de adaptación de la adolescente con respecto a su maternidad. Sin embargo, Stockdale, Crase y Petersen (como se cita en Samuels, Stockdale \& Crase, 1994) han encontrado que la influencia positiva del soporte de la pareja va decreciendo con el tiempo. Probablemente esto está asociado a la relación inestable y precaria que las jóvenes tienen con sus parejas. 


\section{Objetivo del estudio de caso}

Consiste en describir y comparar las representaciones maternas y las interacciones con su bebe en dos madres adolescentes.

\section{Metodología}

\section{Participantes}

Fueron dos madres adolescentes de 17 años que llamaremos "Giovanna" y "María". Sus bebes tenían entre dos y cuatro meses al momento de la evaluación y la entrevista. Las madres eran residentes de Villa El Salvador y se atendían en un hospital materno-infantil de la zona. Ambas vivían con sus familias de origen. Giovanna, además, vivía con su pareja (padre biológico del bebe), mientras que María no vivía con su pareja, pero mantenía una relación con él como enamorados.

\section{Instrumentos}

Para este estudio, las representaciones maternales de las madres adolescentes fueron exploradas a través de una entrevista semi-estructurada sobre representaciones maternales que se construyó especialmente para la investigación más amplia (Traverso, 2006). Esta entrevista estuvo basada en el trabajo de Stern (1997) y Ammaniti et al. (1992), y contenía los siguientes temas: niñez de la madre, la relación con su madre, la relación con su padre, el bebe imaginario y el bebe real, la percepción de sí misma como madre, el padre del bebe como pareja y como padre. Las preguntas de la entrevista fueron validadas a través de un análisis de contenido. Nueve jueces independientes y expertos en infancia temprana evaluaron la relevancia y adecuación de las preguntas. Todas obtuvieron un índice de acuerdo del 100\%. 
A partir del análisis de la entrevista semi-estructurada sobre representaciones maternas se crearon sub-categorías que fueron divididas, a su vez, en dos secciones: representaciones maternas propiamente dichas e historia de vida relacionada con las representaciones maternas. Con la finalidad de establecer la validez de contenido de las sub-categorías, nueve jueces expertos - diferentes de los jueces que evaluaron las preguntas de la entrevista - evaluaron la pertinencia de las sub-categorías. Todas las categorías (menos una) tuvieron un índice de acuerdo entre $89 \%$ y $100 \%$. Para evaluar la confiabilidad del puntaje (1 ó 0) asignado a cada sub-categoría, se llevó a cabo un estudio de confiablidad intracodificador e inter-codificador.

Las interacciones madre-bebe fueron evaluadas y filmadas a través del Mother-Infant Global-Ratings (Murray, Fiori-Cowley \& Cooper, 1996). Esta escala analiza interacciones cara a cara entre madres e infantes de dos a cuatro meses de edad. La escala evalúa la cualidad de la conducta maternal en la interacción, la conducta del bebe y la interacción en sí misma. El video es grabado durante cinco minutos en un contexto de juego libre.

\section{Resultados}

\section{Sobre las interacciones}

En el video filmado y evaluado a través de los Global Ratings, Giovanna se muestra con pocos signos de afecto positivo y poca calidez hacia el bebe. No responde o ignora todas las señales de su hijo. Frecuentemente, a través de la interacción, le demanda a su bebe que la mire y que le haga caso. La joven se muestra intrusiva con el infante, y esta conducta causa, finalmente, que él la evite. No obstante, cabe mencionar que se trata de un bebe que, a través de la interacción observada, se muestra poco dispuesto a explorar su ambiente y se encuentra más ensimismado. En ese sentido, sabemos que hay bebes con tempera- 
mentos diferentes ${ }^{3}$ y entre estos los "difíciles de entusiasmar" (Thomas \& Chess, 1977). Una investigación más exhaustiva de esta díada nos permitiría explorar más si este es el caso o si la evitación del infante es una conducta de autorregulación que se ha establecido ante una madre intrusiva.

En general, al parecer se trata de una interacción difícil, poco placentera y satisfactoria para ambos miembros, y donde la sintonía afectiva (Stern, 1985) no es una característica predominante. Como observadores nos sentimos incómodos y angustiados al mirar esta interacción. Nos identificamos con un bebe que no es reconocido o sostenido y que más bien se siente agobiado por la demanda de su madre.

María, en cambio, se muestra muy cálida en la interacción: su voz y sus gestos expresan afecto y cariño hacia el bebe. No critica ni ignora al bebe; más bien responde a todos sus signos faciales y a sus vocalizaciones. Si bien ella es demandante, en alguna medida, hacia el bebe, estas demandas están hechas de manera apropiada. El bebe está atento a su mamá y mantiene una activa comunicación con ella; es un bebe alegre y vital. Como observadores nos sentimos cómodos con esta interacción porque hay placer, satisfacción y un buen contacto entre ambos miembros.

\section{Sobre las representaciones de sus bebes y de ellas como madres}

Giovanna, describiendo a su bebe, refiere que "él es muy resentido, rencoroso. Algunas veces yo estoy ocupada y él empieza a llorar, quiere su teta, yo lo dejo llorando porque estoy ocupada”. Pareciera que Giovanna proyecta los aspectos más negativos de sí misma sobre el bebe, de manera que estas representaciones pueden funcionar como un obstáculo para leer o interpretar a su hijo o, en términos de Fonagy, Gergely,

3 El tema del temperamento es polémico. Para algunos investigadores, este no existe y es producto de proyecciones y atribuciones de los padres. Desde el psicoanálisis, vemos enfoques como el de Melanie Klein, quien si bien no habla de temperamento sí refiere que hay infantes con diferentes montos de instinto de muerte y envidia, por ejemplo. 
Jurist y Target (2002), observaríamos a una madre con una función reflexiva empobrecida. Cuando se le pregunta sobre su relación con el bebe, ella nos dice: "sí, yo siento que él me quiere; cuando le doy su leche, cuando le hago jugar, él sonríe, se pone engreído. Imagino que me debe querer". Giovanna no tiene la confianza en que su bebe la quiera, porque su propia ambivalencia hacia él le impide conectarse con lo que él siente y necesita, y le hace temer un rechazo del bebe.

En los momentos difíciles con el bebe Giovanna refiere: "yo le he dado palmaditas en distintas ocasiones porque mucho grita, yo me desespero, a veces me pongo nerviosa". En otro momento nos dice: "cuando él llora me pongo nerviosa, le digo 'cállate, cállate'. Algunas veces siento como [ganas de]... o sea de pegarle, pues". Esta joven pareciera tener dificultad para contener a su bebe porque el llanto de este entra en contacto con las "cosas salvajes" (Raphael-Leff, 2003) o con los "fantasmas de la guardería" (Fraiberg, Adelson \& Shapiro, 2003), es decir, con los elementos primitivos no elaborados de su propia historia personal.

Su auto-percepción como madre revela sobre todo aspectos funcionales en el cuidado: "yo soy una buena madre; cuando él hace su caquita, a veces me da pereza cambiarle, pero lo tengo que hacer”. Quizás su manera de estar con el bebe está más vinculada a tareas concretas, pero este estilo le permite sentir que ella tiene algo que ofrecer a su bebe. Ella supera su flojera y no lo deja sucio.

María, en cambio, nos dice: "él es calmado, no llora como otros bebes, solo protesta cuando no le prestan atención”. En esta viñeta vemos cómo María proyecta sobre el bebe los aspectos más positivos sobre sí misma (el bebe ideal). Ella está atenta a las necesidades, y su capacidad para mentalizar al bebe es adecuada. Sobre su relación con el bebe, refiere: "nosotros dormimos juntos en la misma cama; si él se despierta antes que yo, él me mira, cuando yo abro mis ojos, él me mira con una sonrisa, él ríe y yo le comienzo a hablar”. Observamos, en esta viñeta, cómo María siente que el encuentro armonioso y sintonizado con su 
bebe es posible. Es más: ella siente que el bebe la convoca, que la invita a interactuar.

Quizás la confianza en su capacidad de poder entender qué hay en la "mente del bebe" y, por tanto, de poder sintonizar con sus necesidades hace que María logre contener y manejar un momento difícil con el bebe, como muestra esta viñeta: "cuando él llora, yo solamente agarro el coche; le hago pasear o bien lo cargo, más que todo le hago jugar”.

De sí misma, María nos dice: “yo no soy todavía mamá, estoy aprendiendo, yo soy más cariñosa ahora. Nosotros tenemos amor para esta pequeña cosita que ha crecido dentro de nosotras. Lo quiero bastante". Ella todavía no se ubica como mamá: como toda adolescente, entra y sale de su rol. Tal y como veremos a continuación, quizás también su propia madre dificulta este aspecto. En otra viñeta refiere: "anteriormente era renegona, un poco seria, pero ahora no; más alegre soy, paro alegre. Algunas veces me hace renegar porque él también reniega, eso es lo que no me gusta de mí”. Este fragmento narrativo nos permite ver cómo María tiene la capacidad para mirarse a sí misma y a los aspectos de ella que no le gustan. Lo que se evidencia de algún modo es que ella se da cuenta de que su conducta y sus estados emocionales afectan a su bebe y son a su vez afectados por este.

\section{Sobre las representaciones con sus propias madres}

Con respecto a su madre, Giovanna dice: "algunas veces cuando yo le grito, ella dice: ‘¿por qué le has gritado?’. Ella me grita a mí: 'tú no debes gritarle a él, es tu bebe'. A veces un poco que me estira la mano porque ella no quiere le toque al bebe". Observamos aquí una posible transmisión intergeneracional de patrones de apego con una función reflexiva empobrecida que se traslada de madre a hija y que se traduce en ambas como una incapacidad para contener las partes-bebe de sí mismas y de los otros. Sin embargo, la abuela sí puede reasegurar a 
Giovanna en su rol de madre: "él es tu bebe" —a diferencia de la madre de María, como veremos a continuación-.

Por otro lado, María dice de su mamá: "mi mamá me da buenos consejos, ella lo cuida bastante a él. Muchas veces le dice a él: 'mi hijo, ella es tu hermana', yo le digo: 'no mamá, él es mi hijo'”. María percibe a su madre como alguien conectada con ella y con su bebe, capaz de pensar en ambos. Sin embargo, la abuela rivaliza con su hija en torno del bebe: ¿quién es la madre? No obstante María, a pesar de sus inseguridades - "todavía no soy mamá"-, pareciera que poco a poco va estableciendo los límites y su propia diferenciación frente a su madre.

\section{Sobre las representaciones con sus parejas}

En el caso de Giovanna, sabemos que vive con su pareja (el padre biológico) y con su familia de origen. ¿Vivir con su pareja le permite tener más claro su rol de mamá, así como diferenciarse de su propia madre en comparación con María? Y, por otro lado, ¿̇vivir con la pareja facilita o dificulta la relación con su bebe? En sociedades como la nuestra, en las que el "machismo" todavía es predominante, la crianza del bebe y las tareas del hogar están aún asignadas a la mujer; puede ser este un factor de mayor tensión para una joven adolescente que tiene, por lo tanto, que atender a su pareja y al bebe, y que encargarse de las labores domésticas. De su pareja, Giovanna refiere: "yo siento que él tiene la culpa que yo haya tenido un bebe. Antes del bebe estábamos más unidos. Él lo quiere, él lo carga y le hace dormir, pero eso sí, no le cambia el pañal hasta ahorita". Como hemos visto en fragmentos anteriores, Giovanna tiene una capacidad limitada para reflexionar sobre sí misma y sobre sus estados emocionales. Deposita en su pareja la responsabilidad del embarazo no deseado. Como en muchas madres adolescentes, la presencia del bebe interfiere en una relación poco sólida $\mathrm{y}$ muchas veces precaria. 
En un estudio previo con madres jóvenes de sectores urbanos y en pobreza (Traverso, 2006) encontramos que, debido a carencias afectivas tempranas, las madres que viven con sus parejas sienten más "celos" de que puedan preferir a los bebes por sobre ellas. En María este sentimiento es mucho más claro: "sí lo quiere, lo hace jugar, bastante; él le habla, le acaricia. Algunas veces yo pienso que él lo va a querer más a él que a mí”. Fraiberg (1987), en su trabajo terapéutico con madres adolescentes, refiere que ha observado intensos conflictos de rivalidad fraterna que provienen de la historia personal de la madre y que son revividos en la relación con el bebe, como si este fuese otro hermano más.

\section{Reflexiones finales}

A través del estudio de dos casos de madres adolescentes de la misma edad, se intentó explorar, a través de las representaciones maternas y de las interacciones madre-bebe, dos estilos de estar con (Stern, 1985) el bebe. La maternidad adolescente ha sido y todavía es muy estigmatizada, no solo en nuestro país, sino también en otros contextos sociales. Esta visión dificulta contemplar las maneras que cada madre adolescente tiene de relacionarse con su bebe desde su ciclo vital, su circunstancia y su edad. Como varios estudios demuestran, no existe la "madre adolescente", sino una serie de variables mediadoras que de algún modo son factores de protección de la díada madre-bebe; también encontramos factores de riesgo que dificultan la relación. En nuestro estudio de casos, propusimos explorar más detenidamente — entre muchas otras variables - dos factores: la función reflexiva (mentalización) y las redes de soporte.

La función reflexiva - como mencionamos anteriormente- es la capacidad que tiene la madre para "leer" sus propios estados mentales y los del bebe, aspecto que facilita la sintonía afectiva de la díada. De las dos adolescentes, María es quien tiene más desarrollada esta capacidad, de tal manera que la conducta de su bebe resulta ser predecible 
y significativa. Del mismo modo, su capacidad para entender también sus propios estados mentales y por tanto su modo de comportarse la llevan a mantener un mejor vínculo interno y externo con su infante. Giovanna, en cambio, si bien tiene la disponibilidad para cuidar a su bebe, lo hace de manera más funcional, a través de tareas establecidas de crianza. Pareciera que le es difícil entender y conectarse con lo que está detrás de la conducta de su hijo, es decir, con sus estados mentales. De este modo, el encuentro con su bebe se vuelve angustiante y poco placentero. Giovanna, en vez de mirarse a sí misma y de contener las diferentes fantasías y sentimientos que le suscitan su hijo y su relación con él, proyecta sobre este una serie de características propias que, al hacerlo, resultan persecutorias para su nueva identidad como madre.

En cuanto a las redes de soporte que rodean a las madres, en los dos casos estudiados lo que existe es un "círculo protector", lo cual es muy importante para una madre adolescente que necesita entrar y salir de su rol maternal. No se trata de una madre adulta, figura en la que la fusión primaria con el bebe es lo saludable y esperable: para una adolescente en plena etapa de separación e individuación de sus figuras parentales - especialmente de su madre-, esta fusión podría ser muy amenazante. Es así que necesita que estas redes de soporte la acompañen en su maternidad y le permitan, a manera de un espacio transicional, entrar y salir de su maternaje (Franco, 2005).

En ambos casos la figura de la madre es una presencia de soporte, pero también de conflicto. A Giovanna, esta le señala su rol con claridad —ella es la madre —, aunque también la critica — casi pegándole — por su maltrato hacia el bebe. Repite, de tal modo, estilos de interacción que pasan de una generación a otra sin mayor elaboración ni reflexión. En el caso de María, observamos entre ella y su madre la competencia por ser la figura principal para el bebe. Si bien María le recuerda a su madre que ella (la joven) es la madre del bebe, por otro lado su propia desconfianza respecto de su prematura identidad maternal la coloca en una situación desventajosa frente a la abuela del niño. Además, el 
hecho de que María no viva con su pareja refuerza más aún la lucha de poder por el niño, ya que no existe — al menos en el hogar- la presencia masculina que ponga límites y diferencie la estructura dentro de la nueva familia nuclear.

Para ambas jóvenes, el nacimiento del bebe desestabiliza la ya inestable relación de pareja. Surgen celos, ansiedades y temores de ser desplazadas por el bebe, lo cual imposibilita la fantasía inconsciente de satisfacer carencias infantiles no resueltas a través de la pareja.

\section{Referencias}

Abad, D. (2003). Esta es la villa. Recuperado desde http://www.venus. unive.it.

Ammaniti, M., Baumgartner, E., Candelori, C., Perucchini, P., Pola, M., Tambelli, R. et al. (1992). Representations and narratives during pregnancy. Infant Mental Health Journal, 13(2), 167-182.

Apoyo, Opinión \& Mercado. (2004). Perfiles zonales. Recuperado el 30 de septiembre de 2004, desde http://www.apoyo.com/aom.

Becerra, S. (1995). Factores demográficos y psicosociales asociados a la decisión de continuar o terminar el embarazo en un grupo de adolescentes de estrato socio-económico bajo. Revista de Psicología de la Pontificia Universidad Católica del Perú, 13(1), 51-63.

Brooks-Gunn, J. \& Chase Lansdale, P. (1994). Adolescent parenthood. En H. Marc \& H. Bornstein (Eds.), Handbook of parenting: Vol. 3. Status and social conditions of parenting (pp. 113-150). Hillsdale, NJ: Lawrence Erlbaum.

Buitrón, A. (2003). Identidad y maternidad. Estereotipos de género, maternidad adolescente y barreras ante la planificación familiar. Lima: Asociación Peruana de Salud Mental para la Mujer.

Contreras, C. (2001). Madres adolescentes y entorno familiar. REDESS Jóvenes, 2(3), 2-7. 
Contreras, J., Mangelsdorf, S., Rhodes, J., Diener, M. \& Brunson, L. (1999). Parent-child interaction among Latin adolescent mothers. Journal of Research Adolescence, 9(4), 417-440.

Fonagy, P., Gergely, G., Jurist, E. L. \& Target, M. (2002). Affect regulation, mentalization and the development of the self. Nueva York: Other Press.

Fraiberg, S. (1987). The adolescent mother and her infant. En L. Fraiberg (Ed.), Selected writings of Selma Fraiberg (pp. 166-182). Columbus, OH: Ohio State University Press.

Fraiberg, S., Adelson, E. \& Shapiro, V. (2003). Ghosts in the nursery: A psychoanalytic approach to the problems of impaired infantmother relationships. En J. Raphael-Leff (Ed.), Parent-infant psychodynamics: Wild things, mirrors and ghosts (pp. 87-117). Londres: Whurr.

Franco, C. (1993). La experiencia de Villa El Salvador. Del arenal a logros fundamentales a través de un modelo de avanzada. En B. Kliksberg (Ed.), Pobreza: un tema impostergable (pp. 421-432). México, DF: Fondo de Cultura Económica.

Franco, R. (2005, diciembre). La "matriz maternante": respuestas a la paradoja de la maternidad adolescente. Documento presentado en el XIV Encuentro Latinoamericano Donald W. Winnicott: Trazos y Espacios, Lima, Perú.

Instituto Nacional de Estadística e Informática, Agencia de los Estados Unidos para el Desarrollo Internacional, Fondo de las Naciones Unidas para la Infancia y Measure/DHS+, Macro Internacional INC (2001). Encuesta demográfica y de salud familiar. ENDES - 2000. Lima: INEI.

Koniak-Griffin, D. \& Turner-Pluta, C. (2001). Health risks and psychosocial outcomes of early childbearing: A review of the literature. Journal of Perinatal and Neonatal Nursing, 15(2), 1-17.

Levine Coley, R. \& Chase-Lansdale, L. P. (1998). Adolescent pregnancy and parenthood: Recent evidence and future directions. American Psychologist, 53(2), 152-166. 
Luster, T. \& Dubow, E. (1993). Predictors of the quality of the home environment adolescent mothers provide for their school children. Journal of Youth and Adolescence, 19(5), 475-494.

Luster, T. \& Mittelstaedt, M. (1993). Adolescent mothers. En T. Luster \& L. Okagaki (Eds.), Parenting: An ecological perspective (pp. 6999). Hillsdale, NJ: Lawrence Erlbaum.

Murray, L., Fiori-Cowley, A. \& Cooper, P. (1996). The impact of postnatal depression and associated adversity on early mother-infant interactions and later infant outcome. Child Development, 67, 2512-2526.

Musick, J. (1994). The special role of parenting in the context of poverty: The case of adolescent motherhood. En A. Nelson (Ed.), Threats to optimal development: Integrating biological, psychological and social risk factors. The Minnesota Symposium (pp. 179216). Hillsdale, NJ: Lawrence Erlbaum.

Nóblega, M. (2006). Identidad y maternidad en jóvenes que fueron adolescentes en el distrito de Villa El Salvador. Tesis de licenciatura no publicada, Universidad Peruana Cayetano Heredia, Lima.

Osofsky, J., Hann, D. \& Peebles, C. (1993). Adolescent parenthood: Risks and opportunities for mothers and infants. En Ch. Zeanah, Jr. (Ed.), Handbook of infant mental health (pp. 106-119). Nueva York: Guilford Press.

Pimentel, C. (2000). Salud mental y comunidad: una experiencia en desarrollo. Lima: Centro Comunitario de Salud Mental.

Raphael-Leff, J. (2003). Where the wild things are. En J. Raphael-Leff (Ed.), Parent infant psychodynamics: Wild things, mirrors and ghosts. Londres y Nueva York: Whurr Publishers.

Samuels, V., Stockdale, D. \& Crase, S. (1994). Adolescent mothers' adjustment to parenting. Journal of Adolescence, 17, 427-443.

Smithbattle, L. (2000). The vulnerabilities of teenage mothers: Challenging prevailing assumptions. Advance Nursing Science, 23(1), 29-40.

Stern, D. (1985). The interpersonal world of the infant: A unified view of parent-infant psychotherapy. Nueva York: Basic Books. 
Stern, D. (1997). La constelación maternal. Barcelona: Paidós.

Stevens-Simon, C. \& Mc Anarney, E. (1996). Adolescent pregnancy. En R. DiClemente, W. Hansen \& L. Ponton (Eds.), Handbook of adolescent health risk behavior (pp. 313-332). Nueva York: Plenum Press.

Thomas, A. \& Chess, S. (1977). Temperament and development. Nueva York: Brunner/Mazel.

Traverso, P. (2006). Adolescent mothers in a context of poverty in Peru: Maternal representations and mother-infant interactions. Tesis doctoral no publicada, Katholieke Universiteit Leuven, Bélgica.

Recibido 16 de marzo, 2007 Aceptado 30 de junio, 2007 


\section{APÉNDICE \\ Entrevista semi-estructurada sobre representaciones maternas}

¿Cuál es el nombre del bebe? ¿Por qué escogiste ese nombre?

Etapa del embarazo

¿Cómo así tuviste el bebe?

¿Habías pensado en la posibilidad de tener un bebe?

Afronte de la noticia

¿Cómo así te enteraste?

¿Qué pensaste?

¿Qué fue lo que hiciste?

¿A quién se lo contaste?

\section{Aceptación del bebe durante el embarazo}

¿En algún momento pensaste en no tener al bebe o alguien te aconsejó no tenerlo?

¿Qué pensaba tu pareja acerca del bebe?

¿Qué pensaba tu familia acerca del bebe?

\section{Desarrollo del embarazo}

¿Cómo te sentiste durante el embarazo?

¿Tuviste algún temor durante esta etapa?

\section{Características del bebe imaginario}

¿De qué sexo te imaginaste que iba a ser?

¿Cómo te lo imaginaste físicamente?

¿Cómo creías que podría ser su carácter?

\section{Características del bebe real}

Cuéntame ¿Cómo es tu bebe? (preguntar por físico y carácter)

¿Podrías nombrar cinco características de tu bebe?

¿A quién se parece más tu hijo?

\section{Confrontación del bebe real con el imaginario}

¿Él (ella) fue como te lo imaginaste?

\section{Maternaje}

¿Sientes que el bebe te quiere?

¿En qué momento sientes que estás más cerca de tu bebe?

¿Te gusta jugar con tu bebé? 
Descríbeme, ¿cómo eres tú como mamá? ¿Qué clase de mamá eres? ¿Cómo te describirías como madre? ( 5 características)

¿Sientes que puedes cuidar bien a tu bebe?

¿Te es fácil entender lo que le está pasando?

¿Cómo reaccionas cuando llora?

¿Te es fácil calmarlo?

¿Ha habido alguna vez en que ha llorado mucho y no se calmaba? ¿Cómo te sentías o qué hacías cuando no se podía calmar?

¿Cuando el bebe no se podía calmar, te has sentido enfadada, molesta o enojada con él?

¿En medio de la desesperación le has gritado, dado palmadas o pegado?

Cuando han habido estos momentos difíciles, ¿̧has sentido ganas de que otra persona se encargue o cuide del bebe?

¿Cuáles son las tres emociones más fuertes que sientes cuando estás con tu hijo?

¿Hay algo que te gustaría cambiar de ti como mamá?

¿Cuáles son tus temores para ti como mamá?

¿Cuáles son tus deseos para ti como mamá?

¿Cuáles son tus deseos para el futuro de tu hijo?

¿Cuáles son tus temores en torno del futuro de tu hijo?

¿Qué cosas han cambiado después de su nacimiento?

¿Qué cosas han cambiado en ti como persona?

¿El ser madre en este momento interrumpe en algo tu vida, tus metas?

\section{Matriz de apoyo: niñez y actualidad}

¿Quién fue la persona más importante para ti cuando eras niña? ¿Por qué?

¿Quién es la persona que más te cuidó de niña?

¿Quién te cuidó cuando estabas enferma?

¿Te has separado de tu mamá cuando eras niña?

¿Cuál fue tu reacción frente a esta(s) separación(es)?

Si la respuesta a la primera pregunta de esta sección no se refiere a la madre preguntar: ¿Cómo era tu mamá cuando tú eras pequeña?

Si la respuesta a la primera pregunta de esta sección no se refiere a la madre preguntar: ¿Qué recuerdo(s) me podrías contar entre tú y tu mamá?

¿Cómo te sentías cuando eras niña?

¿Sientes que te faltó cariño?

¿Sientes que alguien te ha hecho daño o te ha hecho sufrir cuando eras niña? 
¿Hay algo que quisieras darle a tu bebe que no te dieron o no tuviste de niña? ¿Cómo fue la relación con tu padre cuando eras niña?

En momentos difíciles, ¿̇a quién recurres? ¿Por qué?

¿Sientes que ahora necesitas más apoyo que antes?

¿Te gusta que te ayuden otras personas en el cuidado del bebe?

¿Te sientes bien con esa ayuda?

Vamos a hablar un poco sobre la relación con tu mamá ahora, ¿̇cómo es tu mamá contigo? (5 características)

¿Qué características de tu mamá no te gustaría repetir con tu hijo?

¿Qué características de tu mamá quisieras repetir con tu hijo?

¿Crees que la cantidad de veces que te ves con tu mamá ha variado desde que ha nacido tu bebe?

¿Tienes la impresión de que ahora que ha nacido tu bebe, recuerdas más a menudo cómo era tu mamá contigo cuando eras niña?

¿Te sientes ahora más cerca o más distante de tu mamá?

¿Crees que ahora que eres mamá comprendes mejor a tu mamá, como madre o como persona?

¿Sientes que tu mamá te apoya en el cuidado de tu bebe? ¿Cómo?

¿Qué cosas dice de ti como mamá (te critica, te desvaloriza, te dice cosas bonitas)?

Vamos a hablar un poco sobre la relación con tu papá ahora, ¿cómo es tu relación actual con él?

¿Qué piensa del bebe?

\section{Padre biológico del bebe}

¿Cómo es tu relación actual de pareja?

¿Hubo algún cambio en tu relación de pareja después del nacimiento del bebe?

¿Qué piensa tu pareja del bebe?

¿Crees que él quiere al bebe?

¿Sientes que él te apoya en su cuidado? ¿Cómo?

\section{Cierre}

¿Cómo te has sentido en el último mes?

¿Cómo te has sentido al contarme todas estas experiencias sobre tu vida? 